
\title{
$\angle$ Research Square \\ Adhesive Ability of Different Oral Pathogens to Various Dental Materials: An in Vitro Study
}

\section{Yan Tu}

Zhejiang University School of Medicine Stomatology Hospital https://orcid.org/0000-0002-0443-7037

Xiaolong Lin

Zhejiang University School of Medicine Stomatology Hospital

\section{Yuan Wang}

Zhejiang University School of Medicine Stomatology Hospital

\section{Shuli Deng ( $\nabla$ dengshuli@zju.edu.cn )}

Department of Endodontics, The Affiliated Hospital of Stomatology, School of Stomatology, Zhejiang University School of Medicine, Hangzhou, Zhejiang, 310006, China;

\section{Original article}

Keywords: Adhesion, antibacterial properties, bacterial biofilm, dental materials, scanning electron microscope

Posted Date: June 1st, 2021

DOl: https://doi.org/10.21203/rs.3.rs-553051/v1

License: (c) (i) This work is licensed under a Creative Commons Attribution 4.0 International License. Read Full License 


\section{Abstract}

This study provides a basis for selecting dental materials and lays a foundation for developing new dental materials. Four dental restorative materials were divided into two groups: Streptococcus mutans and Actinomyces viscosus, five root canal sealing materials were divided into two groups:

Porphyromonas gingivalis and Enterococcus faecalis. Each material block was immersed in the corresponding group of the bacterial solution and cultured under anaerobic conditions at $37^{\circ} \mathrm{C}$ for $2,4,6$, $8,12,16,20$, and $24 \mathrm{~h}$. The adhesion of bacteria was observed, the number of different bacteria adhering to various material model disks was calculated at different time intervals under a scanning electron microscope. 24 hours later, the number of Streptococcus mutans and Actinomyces viscosus that adhered to the surface of the resin and the zinc phosphate cement material disks was the maximum. The number of Porphyromonas gingivalis and Enterococcus faecalis adhering to the surface of the AH Plus sealer block was the maximum. Streptococcus mutans and Actinomyces viscosus exhibited the strongest adhesion ability to the resin and the zinc phosphate cement material block. Porphyromonas gingivalis and Enterococcus faecalis showed the highest adhesion ability to the AH Plus sealer block.

\section{Full-text}

Due to technical limitations, full-text HTML conversion of this manuscript could not be completed. However, the manuscript can be downloaded and accessed as a PDF.

\section{Figures}




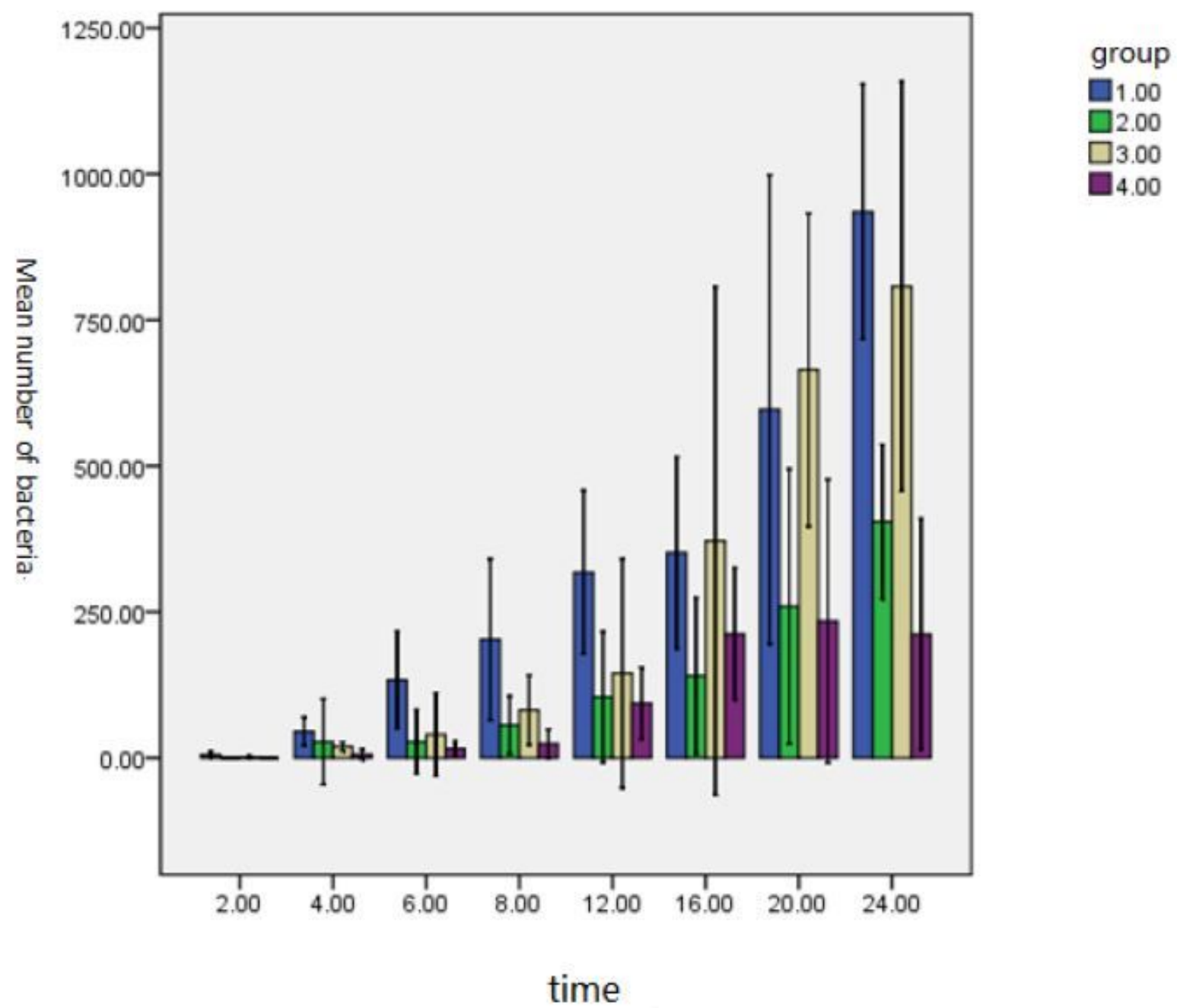

Group: 1. 3M Z350 resin, 2. glass ionomer cement, 3. zinc phosphate cement, 4. Icon; (Bacteria count unit: number $\times 5000$ visual field; Time unit: hour)

Figure 1

Streptococcus mutans group 

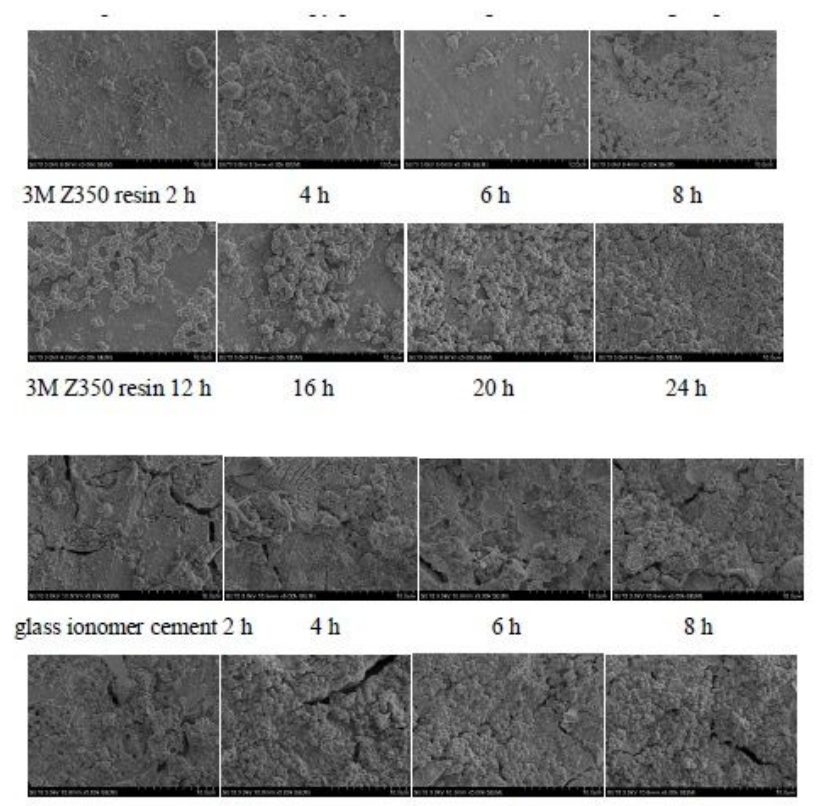

glass ionomer cement $12 \mathrm{~h} \quad 16 \mathrm{~h} \quad 20 \mathrm{~h} \quad 24 \mathrm{~h}$

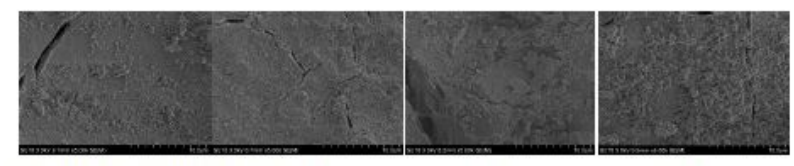

zinc phosphate cement $2 \mathrm{~h} \quad 4 \mathrm{~h} \quad 6 \mathrm{~h} \quad 8 \mathrm{~h}$

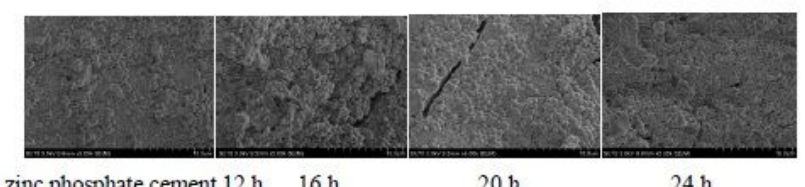

zinc phosphate cement $12 \mathrm{~h} \quad 16 \mathrm{~h}$

$20 \mathrm{~h}$

$24 \mathrm{~h}$
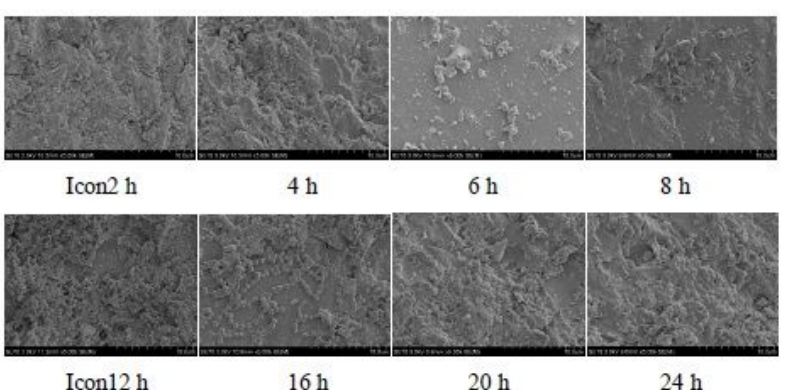

\section{Figure 2}

Electron microscopy pictures of Streptococcus mutans group 


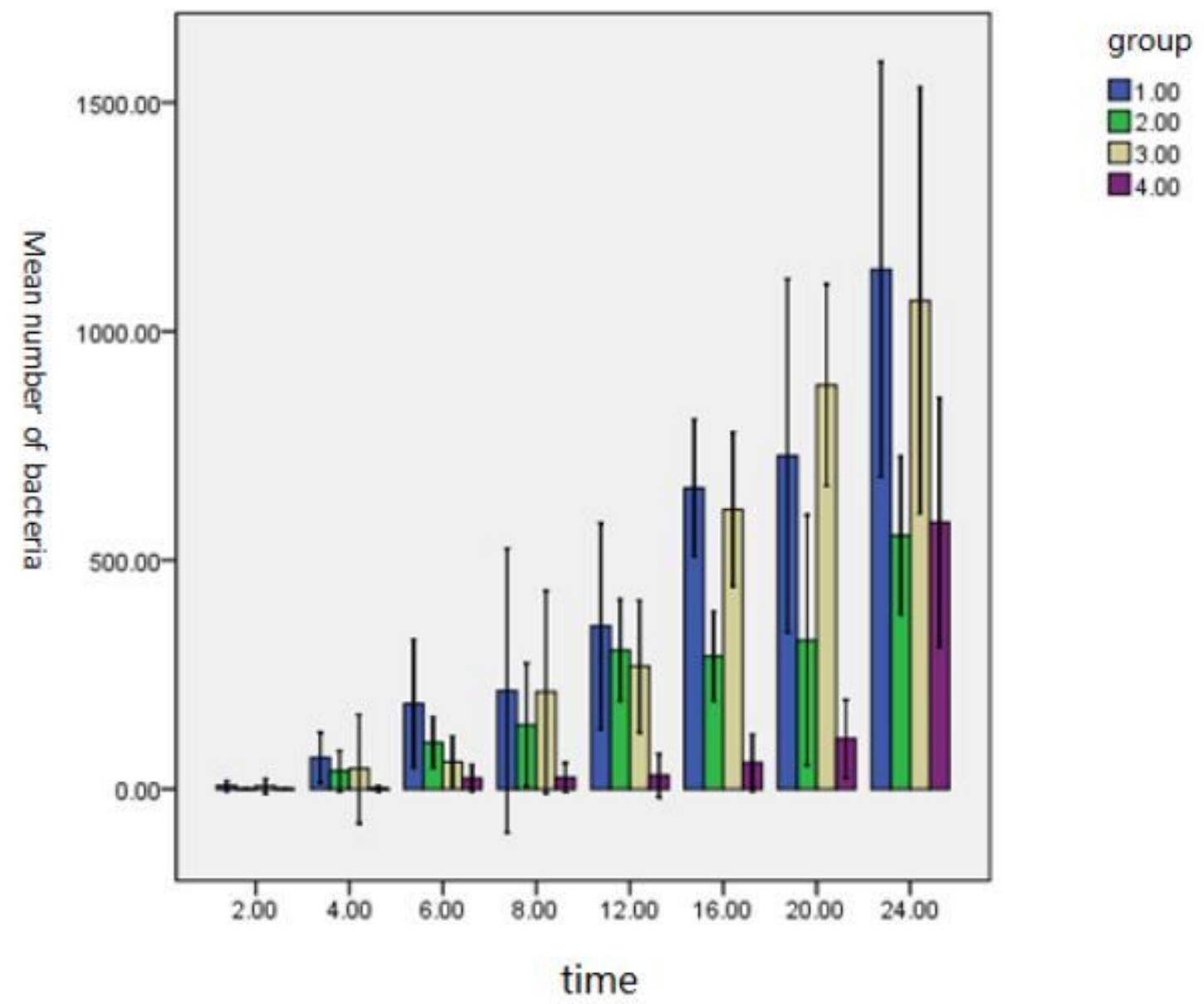

Group: 1. 3M Z350 resin, 2. glass ionomer cement, 3. zinc phosphate cement, 4. Icon; (Bacteria count unit: number $\times 5000$ visual field; Time unit: hour)

Figure 3

Actinomyces viscosus group 

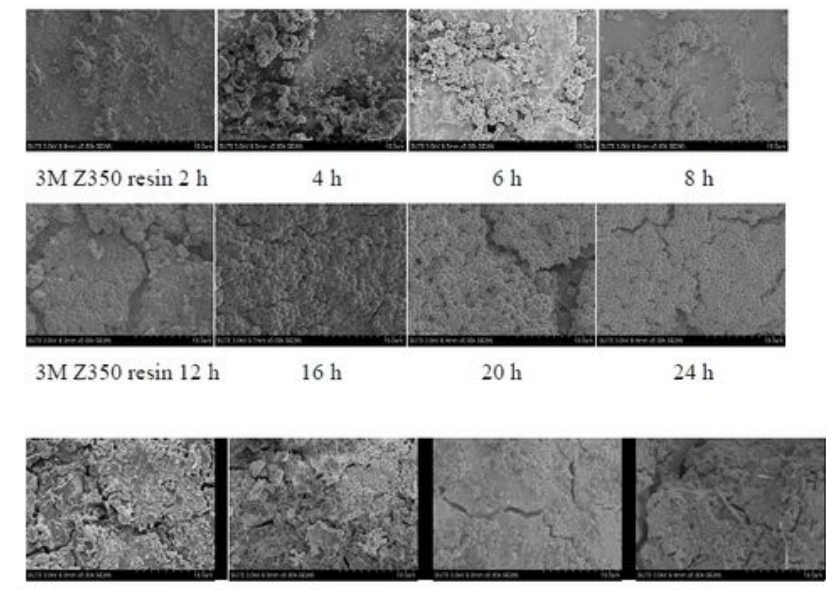

glass ionomer cement $2 \mathrm{~h} \quad 4 \mathrm{~h} \quad 6 \mathrm{~h} \quad 8 \mathrm{~h}$

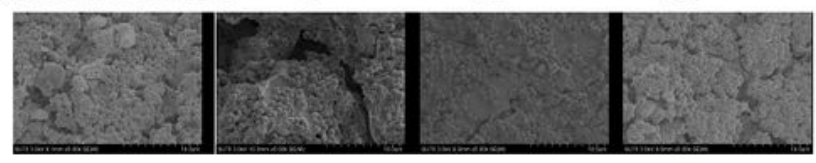

glass ionomer cement $12 \mathrm{~h} \quad 16 \mathrm{~h} \quad 20 \mathrm{~h} \quad 24 \mathrm{~h}$

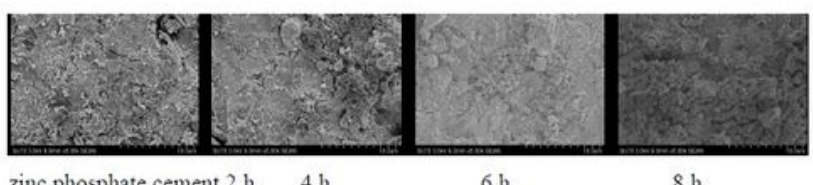

zinc phosphate cement $2 \mathrm{~h} \quad 4 \mathrm{~h} \quad 6 \mathrm{~h}$

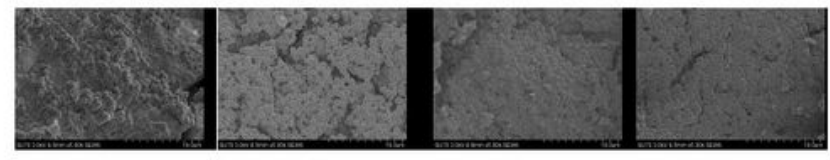

zinc phosphate cement $12 \mathrm{~h} \quad 16 \mathrm{~h}$

$20 \mathrm{~h} \quad 24 \mathrm{~h}$

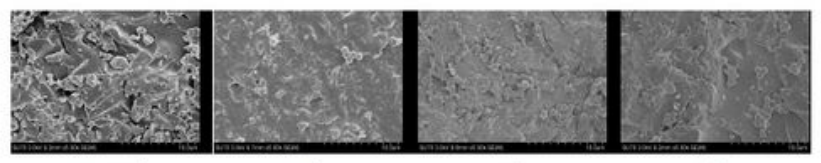

Icon $2 \mathrm{~h}$

$4 \mathrm{~h}$

$8 \mathrm{~h}$

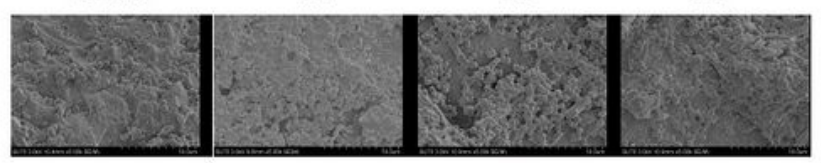

Icon12 h

$16 \mathrm{~h}$

$20 \mathrm{~h}$

$24 \mathrm{~h}$

Figure 4

Electron microscopy pictures of Actinomyces viscosus group 


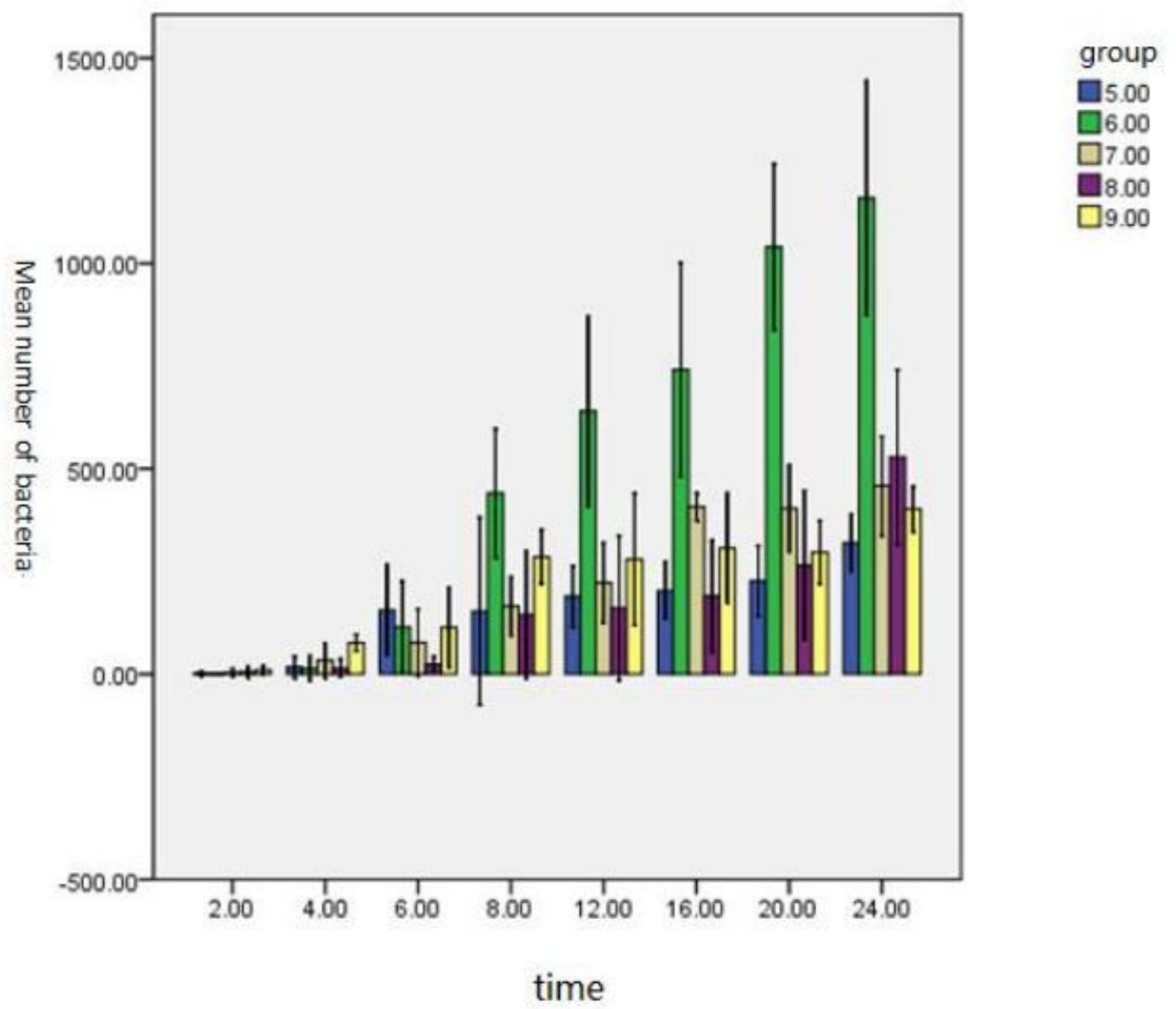

Group: 5. MTA, 6. AH-Plus, 7. iRoot-SP, 8. iRoot-BP, 9. iRoot-FS;

(Bacteria count unit: number $/ \times 5000$ visual field; Time unit: hour)

Figure 5

Porphyromonas gingivalis group 

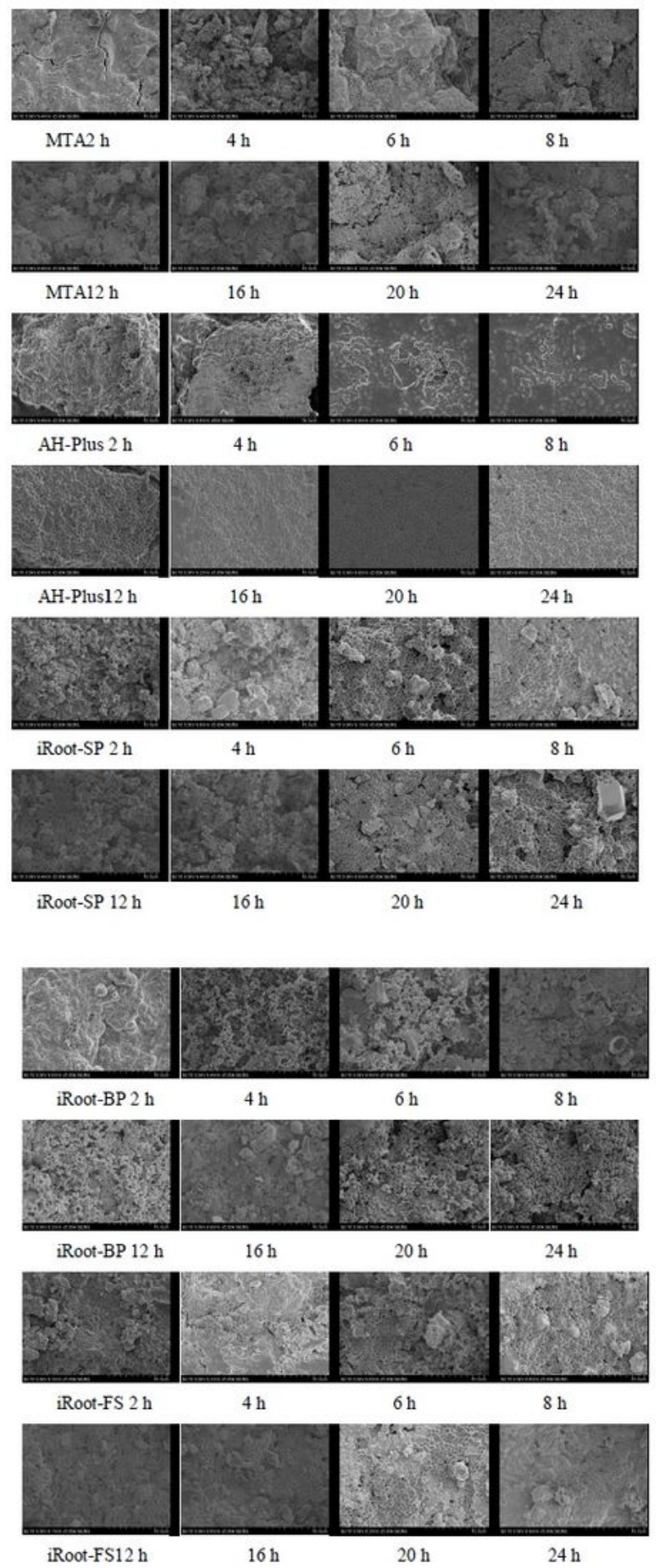

Figure 6

Electron microscopy pictures of Porphyromonas gingivalis group 


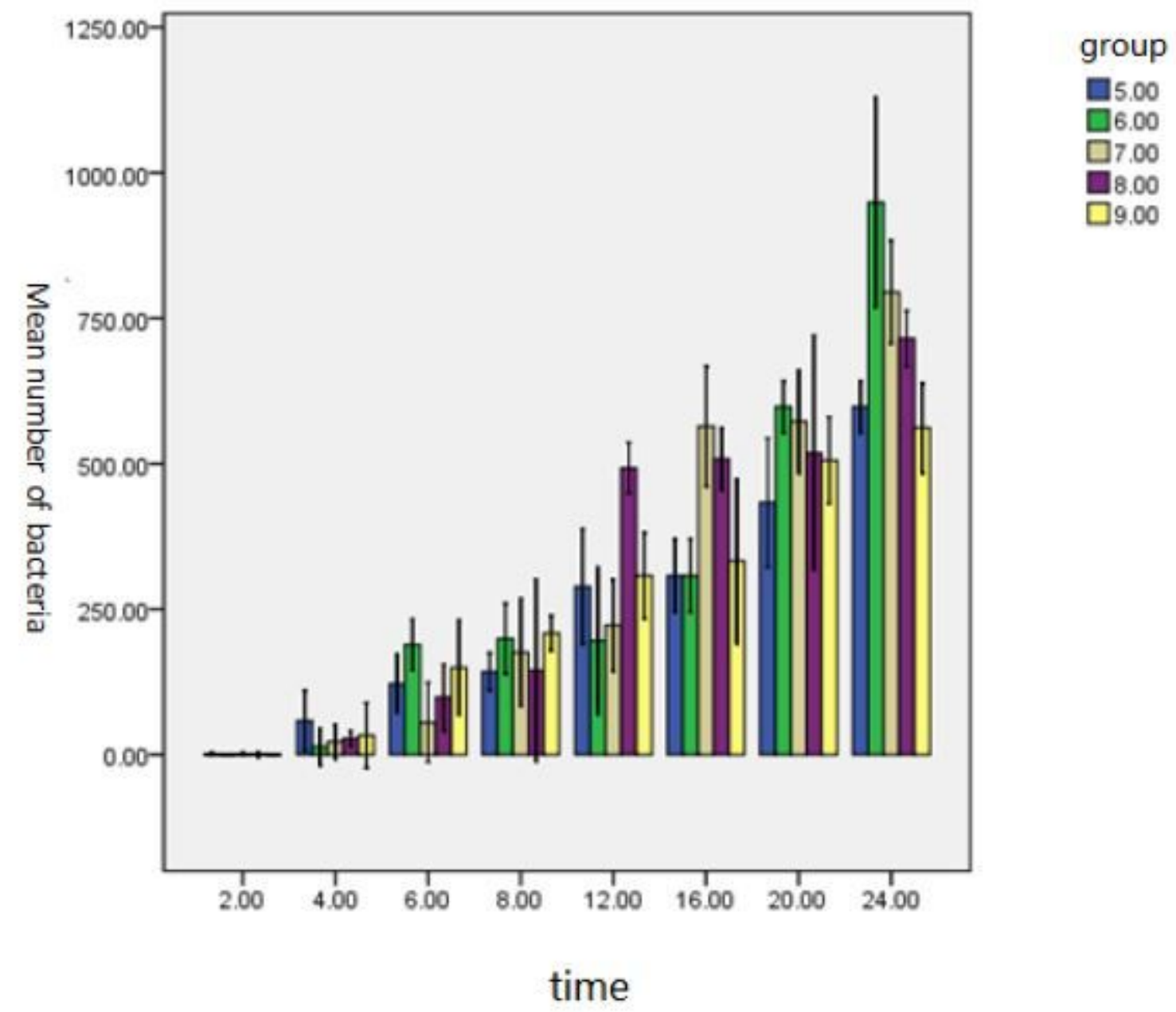

Group: 5. MTA, 6. AH-Plus, 7. iRoot-SP, 8. iRoot-BP, 9. iRoot-FS;

(Bacteria count unit: number $\times 5000$ visual field; Time unit: hour)

Figure 7

Enterococcus faecalis group 

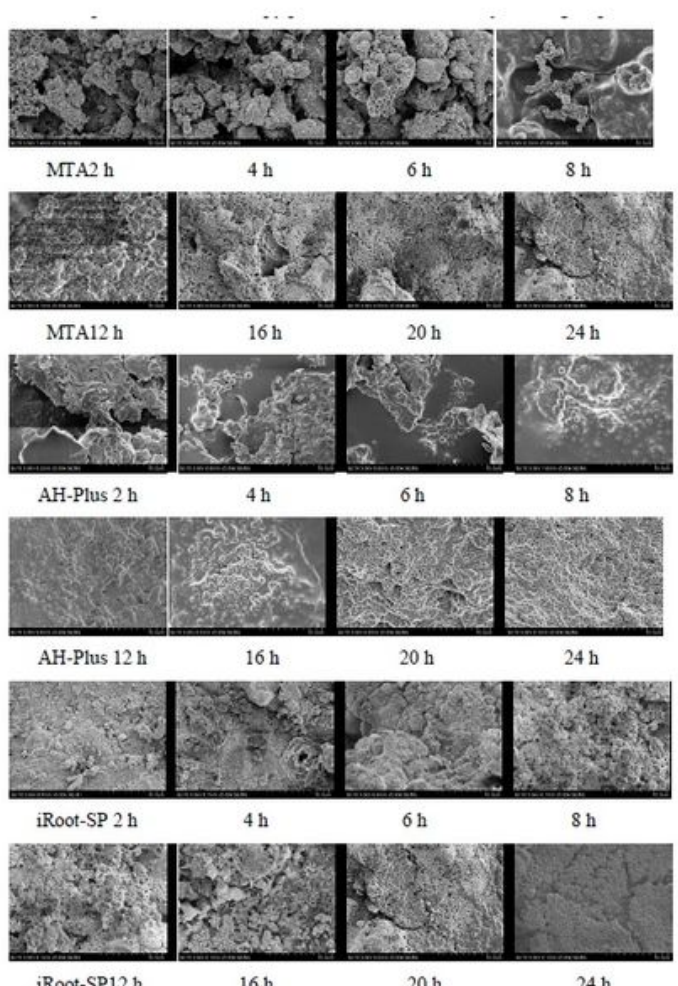

iRoot-SP12 h

$16 \mathrm{~h}$

$20 \mathrm{~h}$
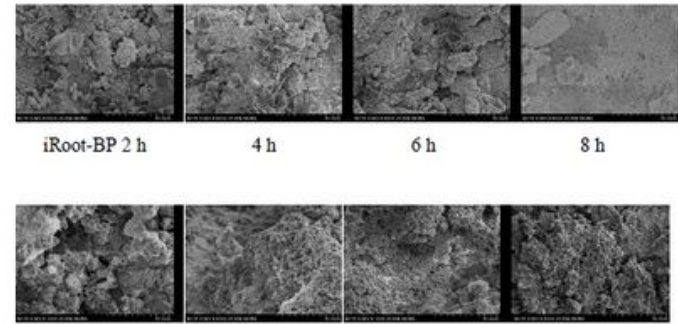

iRoot-BP12 h

$16 \mathrm{~h} \quad 20 \mathrm{~h}$

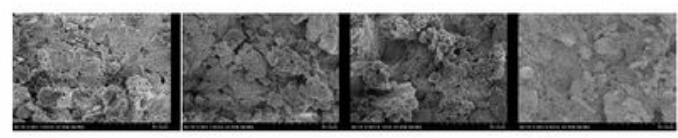

iRoot-FS $2 \mathrm{~h}$

$4 \mathrm{~h}$

$6 \mathrm{~h}$

$8 \mathrm{~h}$

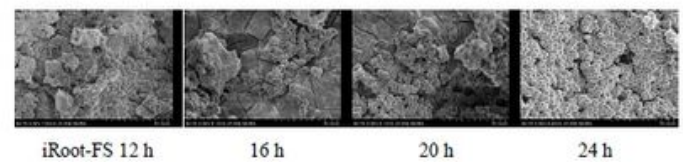

Figure 8

Electron microscopy pictures of Enterococcus faecalis group

\section{Supplementary Files}

This is a list of supplementary files associated with this preprint. Click to download. 
- Table.pdf

Page $11 / 11$ 